\title{
Linear colour correction for multiple illumination changes and non-overlapping cameras
}

\author{
Juan Torres , Klamer Schutte, Henri Bouma , Jose-Manuel Menéndez
}

\begin{abstract}
Many image processing methods, such as techniques for people re-identification, assume photometric constancy between different images. This study addresses the correction of photometric variations based upon changes in background areas to correct foreground areas. The authors assume a multiple light source model where all light sources can have different colours and will change over time. In training mode, the authors learn per-location relations between foreground and background colour intensities. In correction mode, the authors apply a double linear correction model based on learned relations. This double linear correction includes a dynamic local illumination correction mapping as well as an inter-camera mapping. The authors evaluate their illumination correction by computing the similarity between two images based on the earth mover's distance. The authors compare the results to a representative auto-exposure algorithm found in the recent literature plus a colour correction one based on the inverse-intensity chromaticity. Especially in complex scenarios the authors' method outperforms these state-of-the-art algorithms.
\end{abstract}

\section{Introduction}

Retrieving objects seen in earlier images is an active research topic within image processing. In very controlled environments the assumption will hold that the pixel values of an object are comparable between past and present images, but in general this is not the case due to illumination variations. Within this paper we consider conditions with multiple illumination sources, each with a different colour and a space-time variant intensity. In effect, the illumination will be different for every position, time and colour channel. In addition, we consider a non-overlapping multi-camera setup. The challenge addressed in this paper is to correct under such conditions images of the same objects such that their corresponding pixels are comparable, to allow re-identifying those objects along time and space.

Three different strategies can be distinguished in the literature to improve the performance of re-identification algorithms under varying imaging conditions:

1. Control the camera parameters during acquisition, by using auto-exposure (AE) or auto-white balance control.

2. Assume that the matching algorithm handles the imaging condition changes. For instance, using intensity invariant colour descriptors.

3. Correct the images using image processing after acquisition but before applying the matching algorithm.

Table 1 summarizes the main relevant approaches.
The first strategy is widely applied on current available cameras [1, 2]. These methods control the camera parameters during acquisition to compensate for the light variations and obtain suitable images for viewing or processing purposes. Nevertheless, with multiple light sources, each with different spatial effect, such approaches are unable to effectively correct at every position within an image because they do not allow for location-dependent corrections. Liu et al. [3] goes far beyond the classic approach because their division into regions provides better knowledge of the light behaviour, making the correction more accurate.

The second strategy is often based on object descriptors that able to match two images of the same object under different pose and light conditions. Such descriptors [4] may include information about colour, location, structure, texture or a combination of several of these. Using invariant features may reduce the amount of information; for example, in an intensity normalised image - a common descriptor - it will be very hard to separate grey clothes of people from a black background (BG). These kinds of techniques are hence avoided in this paper.

The third strategy, including this work, may be used on its own or in combination with the previously explained. They are mainly based on knowledge about image formation and surface reflectance models and consist of designing computational colour constancy methods that 'estimate the chromaticity of the light source and then correct the image to a canonical illumination using the diagonal model' [23]. This 'diagonal model', introduced by Finlayson et al. [24] as a diagonal-matrix transform (DMT) based on the von 
Table 1 Colour correction approaches

\begin{tabular}{lc}
\hline Based on & References \\
\hline acquisition control & {$[1-3]$} \\
colour descriptors & {$[4]$} \\
colour constancy & {$[5-9]$} \\
camera response function & {$[10-13]$} \\
inter-camera colour response & {$[14-22]$} \\
\hline
\end{tabular}

First row refers to the first strategy and the remainders to the third strategy while the second strategy is not dealt with in this paper

Kries' model, consists of a linear transformation for each of the colour channels. By means of the DMT, the colour appearance under several light conditions can be maintained. A complete survey about colour constancy algorithms can be read in [7]. Nevertheless, these methods have high computational cost and require complex calibrations using known illuminants, causing these techniques to fail when they are unknown. Furthermore, even though there are several attempts to deal with real images, their accuracy [5] - or their application to complex scenarios $[6,8,9]$ - are not sufficiently demonstrated.

Other research lines model the camera response function (CRF) [10] which consists of establishing the relation between the amount of light collected by the camera sensor and the digital intensity values. Using these models, several correction algorithms may be applied by comparing current pixel intensities to reference or previous values. For instance, Withagen et al. [11] developed a simple and fast correction method by presenting different ways to estimate a global intensity correction factor to correct the input images. This method has been recently complemented by Sayed and Delva [13], who added a local correction using the mean and standard deviation variation. Nevertheless, this calculation is very sensitive to noise and introduces blurring artefacts in the image. Inspired by Grossberg and Nayar's work [10], Parameswaran et al. [12] implemented an illumination compensation method based on the fact that the order of the pixel intensities is maintained when the illumination changes. When the illumination change is local, several functions must be estimated, which increases the computational cost. Furthermore, for our purpose, these methods are not insufficient because they do not deal with multi-camera systems.

The classic colour constancy algorithms and CRFs were the basis for the first designs of methods that correct photometric differences in non-overlapping, multi-camera architectures. Basically, the inter-camera colour response (ICCR) estimates [14] are based on the assumption that the appearance of an object under distinct illumination conditions will be the same when their respective cumulative histograms are equal. An initial approach from Javed et al. [15] utilised a supervised method performing a probabilistic principal component analysis using the normalised bin histograms of the objects for obtaining the brightness transfer functions (BTFs) between cameras. Several works have been derived from Javed's. They mainly focused on making the method more robust against illumination changes using several frames of the same person for the estimate [16]; defining improved BTFs [18, 19]; or implementing non-supervised methods [17, 21]. The current ones also include spatiotemporal relationships among cameras $[20,22]$. However, ICCR techniques require complex calibration stages, the use of colour checkers [5] or, in the non-supervised methods, a deeper knowledge of the moving objects.

This paper focuses on a colour correction of foreground (FG) objects (persons) based upon observed variations in the $\mathrm{BG}$ in multi-camera scenarios. This correction is composed of a double mapping. The first mapping corrects the local illumination variations whereas the second compensates the photometric changes between different cameras. Separation between FG and $\mathrm{BG}$ is typically performed by object detection and tracking algorithms, which are out of the scope of this paper ([25] presents a complete review of these methods). The correction process is fast in run-time, and it only requires a training mode that learns inter-camera and $\mathrm{BG}-\mathrm{FG}$ relations using several people samples as we describe in the next sections. It also yields worthy results with real images. Colour calibration or knowledge of the illuminants are not required. Advanced matching techniques are also out of the scope. Nevertheless, we made use of the earth mover's distance (EMD) [26] as a similarity measure for the experiments.

The remainder of the paper is organised as follows. In Section 2, our linear correction model is proposed including both the inter-camera as well as the local corrections. Section 3 details the proposed technique. In Section 4, we describe our experiments which show the good performance of our algorithm in a complex photometric real scenario compared with state-of-the-art AE [3] and colour correction [27] algorithms. Finally, Section 5 summarizes conclusions and suggestions for future work.

\section{Linear correction model}

We start providing our notation and assumptions (Section 2.1). Then, we define our model and adapt it to our requirements (Section 2.2). After that, we propose the double linear correction mapping (LCM) (Section 2.3).

\subsection{Notation and assumptions}

In the paper, we use the notation in Table 2.

Our approach has the following assumptions:

Table 2 Notation used in the paper

\begin{tabular}{|c|c|c|c|}
\hline Symbol & Description & Symbol & Description \\
\hline$\lambda$ & wavelength & $b$ & $\begin{array}{l}\text { referred to BG (as a } \\
\text { superscript) }\end{array}$ \\
\hline$E$ & $\begin{array}{c}\text { spectral irradiance of } \\
\text { a light source }\end{array}$ & $f$ & $\begin{array}{c}\text { referred to } F G \text { (as a } \\
\text { superscript) }\end{array}$ \\
\hline$G$ & $\begin{array}{c}\text { in-camera processing } \\
\text { gain }\end{array}$ & $H$ & $\begin{array}{c}\text { normalised cumulative } \\
\text { histogram }\end{array}$ \\
\hline 1 & $\begin{array}{c}\text { pixel intensity in an } \\
\text { image }\end{array}$ & $\hat{\jmath}$ & I corrected \\
\hline $\bar{l}$ & average value of I & $\Delta /$ & ratio of two values of \\
\hline$i$ & $\begin{array}{l}\text { illumination source } \\
\text { index }\end{array}$ & $j$ & camera index \\
\hline$k$ & colour channel index & $N$ & $\begin{array}{l}\text { number of total } \\
\text { locations }\end{array}$ \\
\hline$n$ & $\begin{array}{l}\text { location index (range } \\
\qquad(-N)\end{array}$ & $Q$ & $\begin{array}{c}\text { camera sensor } \\
\text { sensitivity }\end{array}$ \\
\hline$R$ & $\begin{array}{c}\text { amount of electrons } \\
\text { generated by each } \\
\text { sensor pixel }\end{array}$ & $S$ & $\begin{array}{c}\text { photometric response } \\
\text { of a surface }\end{array}$ \\
\hline$t$ & time & $V$ & $\begin{array}{c}\text { number of total } B G \\
\text { regions }\end{array}$ \\
\hline$v$ & $\begin{array}{l}\text { BG region index } \\
\text { (range } 1-V)\end{array}$ & $\boldsymbol{x}$ & pixel coordinates \\
\hline
\end{tabular}


1. Multiple dynamic sources: All light sources can have different colours and will change over time.

2. Lambertian: The objects are mainly composed by Lambertian surfaces.

3. Static surface response: The photometric surface responses $S$ of an object are not dependent of either the illuminants or the camera response; remain similar with time; and are only dependent on the surface properties.

4. Not crowded scenes: As the correction is based on BG information, very crowded scenes - where sufficient BG is not always observed - are not allowed.

5. Linearity: The intensity is neither under nor over saturated and only linear digital processing is applied (ignoring, e.g. gamma correction). Camera's settings hold constant. Although not every camera obeys these assumptions, many cameras do, as is demonstrated in [28].

6. Independent channels: Changes in the product of the illumination $E$ and the camera response $Q$ is within each band $R, G, B$ independent of the surface reflectivity $S$. We expect this to hold for a wide range of light sources, cameras and materials.

\subsection{Image formation model}

The image formation model of image $I$ is modelled by the CRF

$$
I_{k}(x, t)=\mathrm{CRF}_{k}\left(R_{k}(x, t)\right)
$$

where $R$ is the amount of electrons generated by each sensor pixel. In this context $k$ refers to the three (RGB) colour channels. The definition of $R_{k}$ can be extended to

$$
R_{k}(\boldsymbol{x}, t)=\int_{\lambda} Q_{k}(\lambda) E(\lambda, \boldsymbol{x}, t) S(\lambda, \boldsymbol{x}, t) \mathrm{d} \lambda
$$

where the product $E \cdot S$ is the incident light arriving at pixel coordinates $\boldsymbol{x}$ in the sensor plane and $Q$ is the camera sensitivity (similar to the model in [29]). If we assume that the CRF is linear, and we have independent channels (Assumptions 5 and 6 of Section 2.1), (1) and (2) can be simplified to

$$
I_{k}(\boldsymbol{x}, t)=G(t) S_{k}(\boldsymbol{x}, t) \int_{\lambda} Q_{k}(\lambda) E(\lambda, \boldsymbol{x}, t) \mathrm{d} \lambda
$$

where $G$ models the in-camera processing such as exposure variations and gain. In this expression we see three parts: (i) the product of $G$ and $Q$ which depends on the camera; (ii) $E$ which depends on the source of light, location and scenario; and (iii) $S$ which depends on the observed object surface.

In (2) and (3), the illumination dependent term $E$ is a sum of all $i$ illumination sources, and thus, $E(\lambda, x, t)=$ $\sum_{i} E_{i}(\lambda, \boldsymbol{x}, t)$. For each illumination source, we define a time-varying illumination gain $g_{i}$ and a constant illumination term $E C_{i}$, such that $E_{i}(\lambda, \boldsymbol{x}, t)=g_{i}(t) E C_{i}(\lambda, \boldsymbol{x})$. As such we can rewrite the model in (2) and (3) to

$$
\begin{gathered}
R_{k}(\boldsymbol{x}, t)=\sum_{i} g_{i}(t) \int_{\lambda} Q_{k}(\lambda) E C_{i}(\lambda, \boldsymbol{x}) S(\lambda, \boldsymbol{x}, t) \mathrm{d} \lambda \\
I_{k}(\boldsymbol{x}, t)=G(t) S_{k}(\boldsymbol{x}, t) \sum_{i} g_{i}(t) \int_{\lambda} Q_{k}(\lambda) E C_{i}(\lambda, \boldsymbol{x}) \mathrm{d} \lambda
\end{gathered}
$$

\subsection{Linear correction mapping}

Our goal is to obtain a similar digital image of the same object when the photometric conditions and the cameras change. For BG areas, the photometric surface responses of an object in two temporal instants remains constant $\left(S\left(x, t_{\text {ref }}\right) \simeq\right.$ $S(\boldsymbol{x}, t)=S(\boldsymbol{x})$, Assumption 3 of Section 2.1). Therefore two images $I_{j_{\text {ref }}}$ (from camera $j_{\text {ref }}$ ) and $I_{j}$ (from camera $j$ ) of the same $\mathrm{BG}$ area $S_{0}$ having different photometric conditions can be written (3) as

$$
\left.\begin{array}{c}
I_{k, j_{\mathrm{ref}}}\left(\boldsymbol{x}, t_{\mathrm{ref}}\right)=G_{j_{\mathrm{ref}}}\left(t_{\mathrm{ref}}\right) S(\boldsymbol{x}) \int_{\lambda} Q_{k, j_{\mathrm{ref}}} E_{j_{\mathrm{ref}}}\left(\lambda, \boldsymbol{x}, t_{\mathrm{ref}}\right) \mathrm{d} \lambda \\
I_{k, j}(\boldsymbol{x}, t)=G_{j}(t) S(\boldsymbol{x}) \int_{\lambda} Q_{k, j} E_{j}(\lambda, \boldsymbol{x}, t) \mathrm{d} \lambda
\end{array}\right\} \rightarrow
$$

$\Delta I_{k, j}(x, t)$ is the $k$-band relation factor between a reference camera $j_{\text {ref }}$ and time $t_{\text {ref }}$ with a camera $j$ in time $t$.

A corrected image $\hat{I}_{k, j}$ is written as

$$
\hat{I}_{k, j}(x, t)=\Delta I_{k, j}(x, t) \cdot I_{k, j}(x, t)
$$

If the suitable $\Delta I_{k, j}(x, t)$ is found, it is possible to correct any image $I_{k, j}(\boldsymbol{x}, t)$ to make it similar to a reference one $I_{k, j_{\text {ref }}}\left(x, t_{\text {ref }}\right)$.

We define a double LCM for camera $j$ that compensates the variations of $G \cdot Q$ and $E$ in an acquired image $I_{k, j}$ as

$$
\Delta I_{k, j}(\boldsymbol{x}, t)=a_{k, j}^{\mathrm{cam}}(t) \cdot a_{k, j}^{\mathrm{il}}(\boldsymbol{x}, t)
$$

Each image will be corrected by two terms: $a_{k, j}^{\text {cam }}$ is the 'inter-camera correction mapping' (ICCM) that compensates the scenario and change between cameras, and $a_{k, j}^{\mathrm{il}}$ is the 'illumination correction mapping' (ICM) that corrects the change in location within the same camera and illuminant(s).

The variation of $\mathrm{BG}$ intensities $\left(\Delta I_{k, v}^{\mathrm{b}}\right)$ regarding a reference time $t_{\text {ref }}$ is measured using the following expression

$$
\Delta I_{k, v}^{\mathrm{b}}(\boldsymbol{x}, t)=\frac{\bar{I}_{k, v}^{\mathrm{b}}\left(\boldsymbol{x}, t_{\mathrm{ref}}\right)}{\bar{I}_{k, v}^{\mathrm{b}}(\boldsymbol{x}, t)}
$$

where $\bar{I}_{k, v}^{\mathrm{b}}(\boldsymbol{x}, t)$ is the average intensity value of BG region $v$ in location $x$, time $t$ and channel $k$ according to the selection explained in the Section 3.1.1. Note the similarities between (10) and (7).

Assuming camera exposure values are not modified to maintain a linear relation between light and image intensity, the variations of the $\mathrm{BG}$ regions model the terms $g_{i}(t)$ of (5) with respect to $t_{\text {ref }}$ that influence the FGs. For that purpose we estimate the ICM using

$$
a_{k, j}^{\mathrm{il}}(\boldsymbol{x}, t)=\left(1, \Delta I_{k, 1, j}^{\mathrm{b}}(\boldsymbol{x}, t), \ldots, \Delta I_{k, Y, j}^{\mathrm{b}}(\boldsymbol{x}, t)\right) \cdot \boldsymbol{m}_{x, j}
$$

where $\boldsymbol{m}_{x, j}$ comes from (16).

Regarding ICCM, better results are obtained when it is included an offset term capable of partially compensating for model derivations such as camera noise, intensity offsets 
or camera non-linearity. This term accounts for any error because of the simplifications we made regarding the image formation model (Section 2.2). Therefore the original (8) is replaced by the following

$$
\hat{I}_{k, j}(x, t)=\alpha_{k, j}^{\mathrm{cam}} \cdot a_{k, j}^{\mathrm{il}}(\boldsymbol{x}, t) \cdot I_{k, j}(x, t)+\beta_{k, j}^{\mathrm{cam}}
$$

where $\alpha_{k, j}^{\mathrm{cam}}$ and $\beta_{k, j}^{\mathrm{cam}}$ are constants depending on camera $j$.

\section{Method description}

To estimate the relationship between the people response (FG) and the light variations in the rest of the scene (BG), we define a training mode (Section 3.1) and run-time mode (Section 3.2).

\subsection{Training mode}

In training mode, the relation between cameras as well as the relation between changes in $\mathrm{BG}$ and $\mathrm{FG}$ are learned. We establish these relations by using some FG objects seen in different cameras (for ICCM), different locations and illuminations (for the ICM). In Section 3.1.1 we discuss the selection of locations and the segmentation of $\mathrm{BG}$ regions, in Section 3.1.2 we explain how to estimate the ICCM $\left(a_{k, j}^{\text {cam }}\right)$ and in Section 3.1.3 the vectors $\boldsymbol{m}_{x, j}$ for ICM which relates the $\mathrm{FG}$ to the $\mathrm{BG}$.

\subsubsection{Locations and BG region selection: We model} the three-dimensional world geometry in the acquired image for each camera by splitting the scene in a set of $n=\{1, \ldots$, $N\}$ pre-defined locations. Thus, each pixel $\boldsymbol{x}$ is mapped to a location $n$. We define the $\mathrm{BG}$ regions $v=\{1, \ldots, V\}$ associated to each location according to the geometry of the scene. The BG regions ('Reg' in the example of Fig. 1) are used to estimate the change in illumination to correct the $\mathrm{FG}$ objects. It is desirable to select $\mathrm{BG}$ regions that are most influenced by light changes. For example, in Fig. 1 some regions are located on the wall on the left because outdoor light goes through the opposite doors. Thus, the light reflected on this surface will change in accordance with the outdoor light. In our experiments, the BG regions were manually specified.

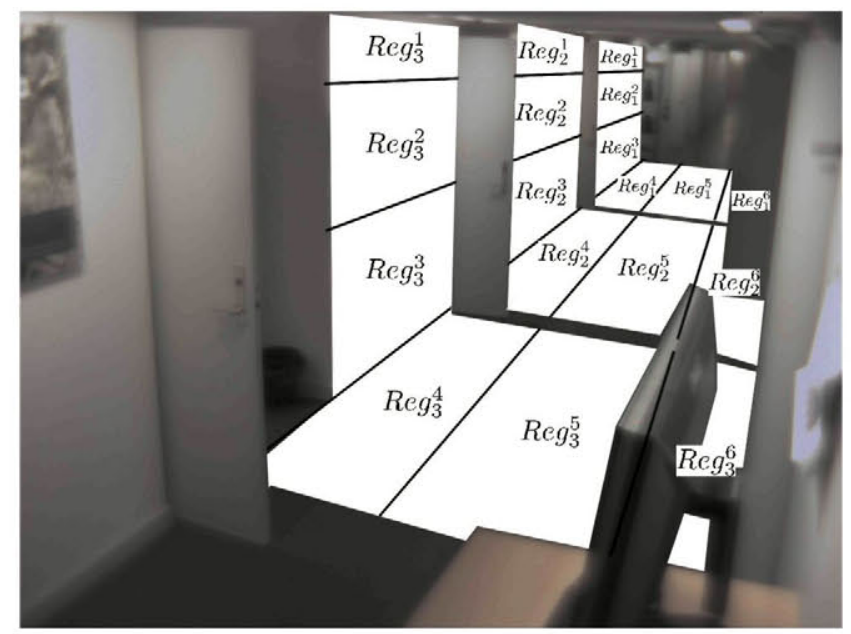

Fig. 1 Indoor surveillance scenario modelling with selected $B G$ regions

Subscript refers to location and superscript to region index
3.1.2 Camera-relation training for ICCM: In the ICCM, we obtain a linear mapping from one camera to another by using several intensity values of FG objects in the different locations to approximate it to the reference one. A comprehensive survey on FG-BG separation is presented by Bouwmans [25]. In our experiments, we use manually segmented FG objects. For comparing two images from two cameras, we make use of the normalised cumulative object histograms $H$ with $h$ values. If there are $\kappa$ training objects, we have $W=h \cdot \kappa$ samples for each location and each colour channel, different from the reference one. Thus, the ICCM of (12) is obtained by minimising the error between the histogram values of the reference camera $j_{\text {ref }}$ and the estimated camera $j$

$$
\begin{aligned}
& \left\{\alpha_{k, j}^{\mathrm{cam}}, \beta_{k, j}^{\mathrm{cam}}\right\} \\
& =\underset{\left\{\alpha_{k, j}^{\mathrm{cam}}, \beta_{k, j}^{\mathrm{cam}}\right\}}{\arg \min } \sum_{w}^{W}\left\|H_{w, k, j}^{\mathrm{f}}-\left(\alpha_{k, j}^{\mathrm{cam}} \cdot H_{w, k, j}^{\mathrm{f}}+\beta_{k, j}^{\mathrm{cam}}\right)\right\|_{2}
\end{aligned}
$$

where $H_{w, k, j}^{\mathrm{f}}$ is the sample $w$ of the normalised cumulative histogram between 0 and 1 of the channel $k$ of the FG in camera $j$. Owing to overexposed pixels, shadows, erroneous $\mathrm{BG}$ region assignments etc., many of these values may be outliers. Since linear least-squares estimates do not work properly when the error distribution is not normal, they are not suitable tools in this case. Instead, we use a robust linear regression algorithm which is able to manage this kind of outliers. It is based on a maximum likelihood estimation [30] which performs an iterative reweighted linear squares regression using a bisquare weight function.

3.1.3 FG/BG-relation training for ICM: The normalised cumulative histograms $H$ are also employed in the estimate of the relationship between $\mathrm{BG}$ and $\mathrm{FG}$. In this case, we extract data from the BG and FG when the light changes. Similar to the ICCM estimate, we have $W=3 \cdot h \cdot \kappa$ samples of data for each location. The estimation is independent of the channel because the relationship models $g_{i}$ are the same in every channel. Using these data, we construct the following ratios expressing the variation for $\mathrm{FG}$ (14) and $\mathrm{BG}$ (15) histograms relative to the reference time $t_{\mathrm{ref}}$

$$
\begin{aligned}
\Delta H_{w, j}^{\mathrm{f}}(\boldsymbol{x}, t) & =\frac{H_{w, j}^{\mathrm{f}}\left(\boldsymbol{x}, t_{\mathrm{ref}}\right)}{H_{w, j}^{\mathrm{f}}(\boldsymbol{x}, t)} \\
\Delta H_{w, v, j}^{\mathrm{b}}(\boldsymbol{x}, t) & =\frac{H_{w, v, j}^{\mathrm{b}}\left(\boldsymbol{x}, t_{\mathrm{ref}}\right)}{H_{w, v, j}^{\mathrm{b}}(\boldsymbol{x}, t)}
\end{aligned}
$$

where $H_{w, j}$ is the sample $w$ of the normalised cumulative histogram of camera $j$ and $v$ refers to the BG region. We fit a linear combination of the BG variations to FG using $\boldsymbol{m}_{x, j}$. This is a vector that relates the change in $V$ BG regions to that in the FG at location $x$, constant through time and equal for each channel $k$ in camera $j$. It is estimated by

$$
\hat{m}_{x, j}=\underset{\tilde{m}_{x, j}}{\arg \min } \sum_{w}^{W}\left\|\Delta H_{w, j}^{\mathrm{f}}-\left(1, \Delta H_{w, 1, j}^{\mathrm{b}}, \ldots, \Delta H_{w, V, j}^{\mathrm{b}}\right) \cdot \boldsymbol{m}_{x, j}\right\|_{2}
$$

where $W$ is the number of samples and $V$ is the number of $\mathrm{BG}$ regions, such that $v=[1, \ldots, V]$. To avoid unreliable samples, 
we eliminate occluded and shadowed areas of the BG. In our experiments, we tested two optimisation methods: robust linear regression algorithm and linear programming. We use the second method as it yields lower errors according to indicators explained in Section 4.2. Nevertheless, the selection of the correct fitting method may depend on the data. In our experiments we observed that, to obtain good fitting, we need enough samples $(W \gg V)$.

\subsection{Run-time mode}

In the run-time mode, the LCM of (12) is applied. It first requires the estimate of the $\mathrm{BG}$ intensities in the image and then the computation of the mappings ICM $\left(a^{\text {il }}\right)$ and ICCM $\left(\alpha^{\mathrm{cam}}, \beta^{\mathrm{cam}}\right)$. Regarding the ICCM, (12) is applied using the parameters estimated by (13).

\section{Experimental evaluation}

\subsection{Experimental setup}

The proposed method was tested in a dedicated experiment with multiple cameras, multiple people, multiple locations and changing lighting conditions. Although there are several datasets on the internet related to people re-identification in a multi-camera network (QMUL underGround Re-IDentification (GRID) dataset. http://www. eecs.qmul.ac.uk/ ccloy/downloads_qmul_underground_reid. $\mathrm{html}$ ) and some others also include light changes (VIPeR: Viewpoint Invariant Pedestrian Recognition. http://vision. soe.ucsc.edu/node/178), (Person Re-ID (PRID) 2011 dataset. http://rs.icg.tugraz.at/datasets/prid/index.php), we were not able to find any dataset that fits all our requirements (overall, BG information and camera's auto-settings off). Therefore we used our own dataset (Multi-Camera Dynamic Light (MCDL) dataset is publicly available in: https://www.researchgate.net/publication/26446 2014_MCDL_Dataset). For the experiment, we used two Firewire cameras influenced by indoor and outdoor illumination. The illumination was changed by switching the indoor lights on and off, by opening and closing doors and by outdoor lighting variations because of changing cloud coverage (see Fig. 2). The time between each capture is enough to have a noticeable change in outdoor lighting. For both cameras, the variation in the average value of images exceeds $10 \%$. In each camera view, we defined three locations (Fig. 1), used one person for the training phase and eight other persons for the test phase. All persons are captured walking in two directions. Each one of the training and test samples were recorded under different outdoor light conditions. In camera 2 , the average $\mathrm{BG}$ intensity during acquisition of the training samples ranges between 102 and 123, whereas the average BG intensity during testing ranges between 97 and 112. The test persons each wore different shirts whose colours are well-distributed along the RGB space. An example picture of each subject is shown in Fig. 3. People outlines are manually segmented to obtain $\mathrm{FG}$ information, used during training and evaluation. The shadows also are manually segmented and discarded. To demonstrate the performance of the method with less optimal segmentation, an experiment has been carried out by eroding and dilating the manually segmented outlines (Section 4.6). A total of 96 frames have been used in training and 192 frames for testing. The 96 training frames have 8 repetitions for each light condition, location and camera ( 8 frames $\times 2$ light conditions $\times 3$ locations $\times 2$ cameras). The 192 test frames have 2 repetitions for each person, light condition, location and camera ( 8 persons $\times 2$ directions $\times 2$ light conditions $\times 3$ locations $\times 2$ cameras).

Camera's AE (aperture, exposure time and gain), auto-white balance (correction between bands) and gamma correction have been disabled.

\subsection{Similarity and error measure}

We evaluate the intensity correction by measuring the dissimilarity between persons using their histograms. We choose the EMD [26], normalised between 0 and 1, a cross-bin algorithm which provides a minimum cost solution to transform one image distribution into another and is robust against noise and small intensity variations. We calculate the Euclidean EMD for each channel and the combined value as the average value of these three EMDs.

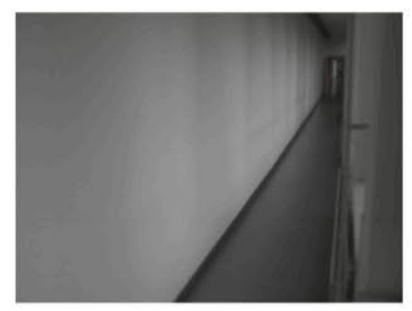

a

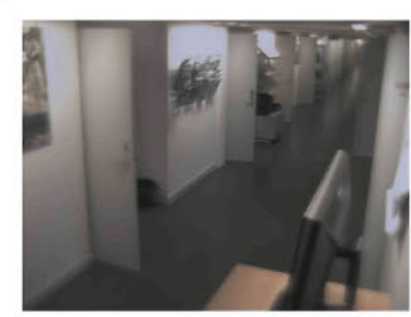

d

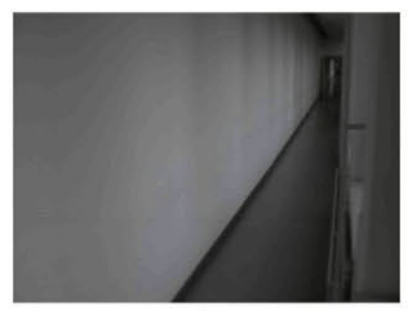

$b$

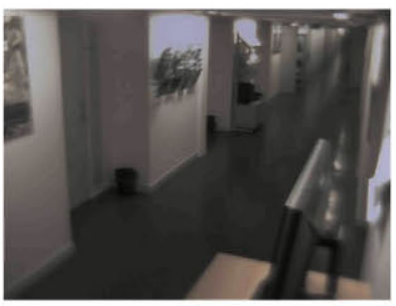

Fig. 2 Examples of light changes in both experimental scenarios Images show variations because of: natural outdoor light variations [(a) and $(b)]$; switch the indoor lights on $(c)$; and open $(d)$ and close $(e)$ multiple doors in the indoor scenario 

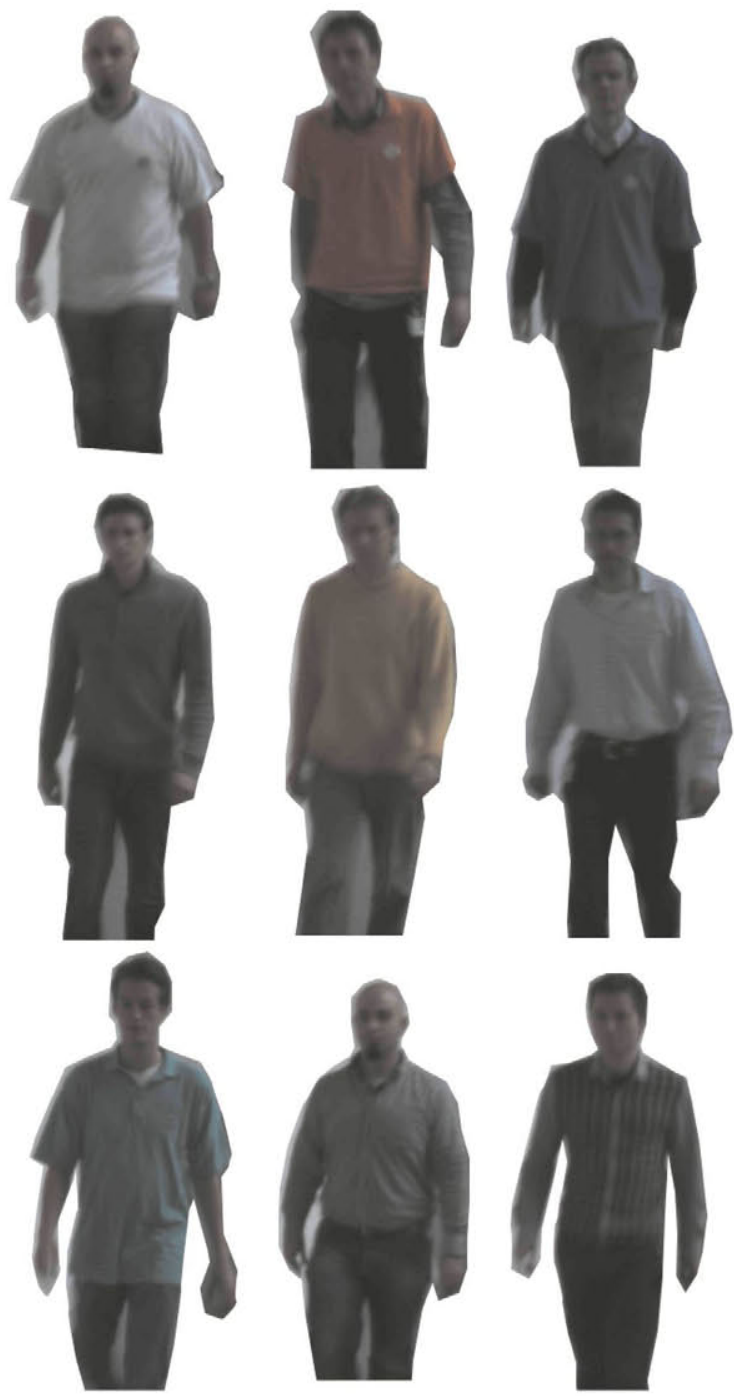

Fig. 3 Example images of each person

The top-left is used for training

We calculated both the intra-class and inter-class distances. For intra-class, denoted with suffix $i i$, we compare between the same person in the different acquisitions: several locations, illumination conditions and cameras. For inter-class, denoted with suffix $i j$, any person is compared with the other people under the same and different photometric conditions.

We evaluate the performance of the system with an error measure that is based on normalised cumulative histograms distribution (CHD) of the computed EMDs. For the intra-class $\mathrm{CHD}_{i i}$ closer to 0 are better whereas for the inter-class $\mathrm{CHD}_{i j}$ closer to 1 are better. Ideally, our algorithm increases the distance between $\mathrm{CHD}_{i i}$ and $\mathrm{CHD}_{i j}$ as much as possible. Using the cumulative histograms, for any threshold value $n_{\mathrm{th}}: \mathrm{FP}\left(n_{\mathrm{th}}\right)=\mathrm{CHD}_{i j}\left(n_{\mathrm{th}}\right)$ and $\mathrm{FN}\left(n_{\mathrm{th}}\right)=$ $1-\mathrm{CHD}_{i i}\left(n_{\mathrm{th}}\right)$, we search for that threshold where the minimal error criterium $\mathrm{MEC}=\mathrm{FP}+\mathrm{FN}$ is minimal.

For this MEC, high error rates are expected because of pose variations, partial occlusions, inter-reflections, self-casting shadows, which all cause the persons to appear differently. The research in this paper only corrects for changes in colour distributions caused by light variations.

The receiver operating characteristic (ROC) curve is used to visualise the performance, where the true positive rate is $\mathrm{TP}(r)=1-\mathrm{FN}(r)$.

\subsection{Implementation of the reference methods}

The obtained results are compared with four cases: direct measurements without any correction, a state-of-the-art automatic exposure algorithm (AE) [3], a colour correction based on the inverse-intensity chromaticity (IIC) [27] and a combination $\mathrm{AE}+\mathrm{IIC}$.

To allow a fair comparison and use the same input images for all methods, the $\mathrm{AE}$ algorithm estimates a correction coefficient based on the brightness of the image, without modifying the camera gain and exposure time. It is based on three parameters: the reference average value to be reached, the number of regions and the weight values of each region. The reference value is calculated by estimating the average value of the illumination reference images of the test set. Liu did not specify the method to set up the number of regions or the weight values. These choices depend on the scenario and how it responds to the light changes. We decide to split the scenario into three regions as it is shown in Fig. 4. Regarding the weights, we choose $0.15,0.7$ and 0.15 from left to right for camera 1 . We give a higher weight to the central region because people are placed there. In a similar way, the chosen weights for camera 2 are $0.2,0.7$ and 0.1 . In this case, the region on the left has a greater weight than the region on the right

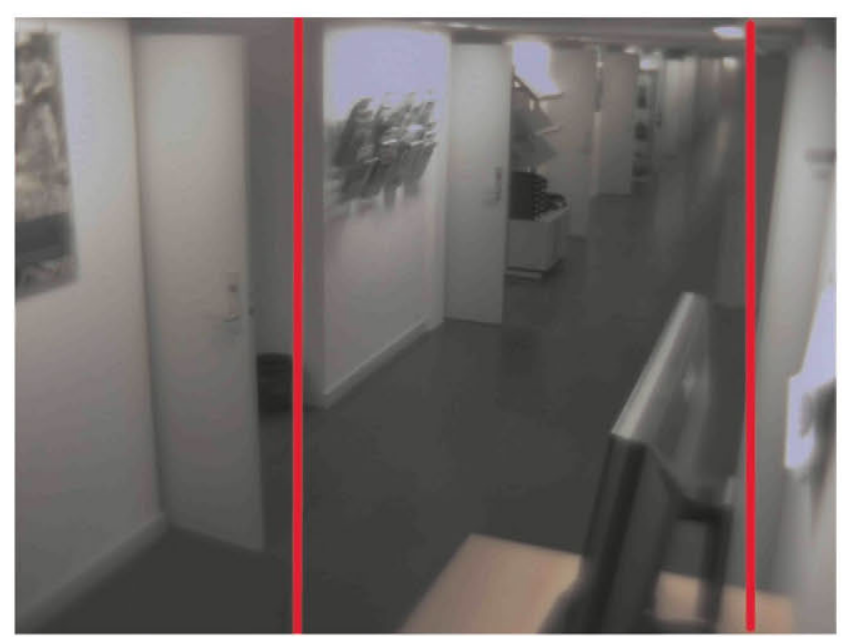

Camera 1

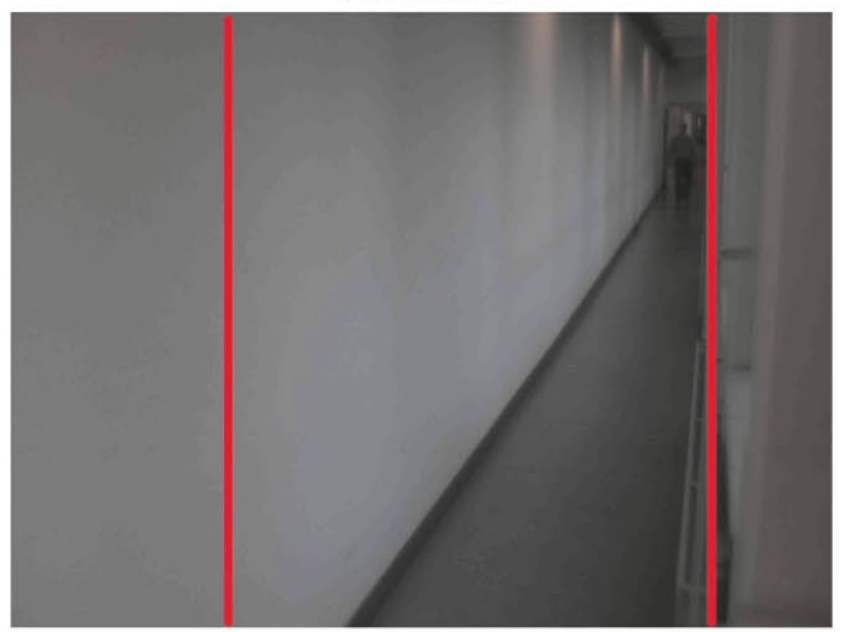

Camera 2

Fig. 4 Region setup used by the AE algorithm described in Section 4.3 
because the light coming from outside is reflected on the wall on the left. These weights have been chosen empirically after testing several variations, being the ones that perform the best.

Similar to AE, IIC is applied to each channel using Tan's implementation. With $\mathrm{AE}+\mathrm{IIC}$, IIC correction is applied over the AE-corrected image.

\subsection{Intra-camera results}

An example of a corrected image for the intra-camera correction is shown in Figs. $5 a, b$ and $d$. Table 3(a) and (b) shows the numerical results of intra-camera experiments. The results from camera 1 show an error reduction for our algorithm compared with the uncorrected case (MEC is 0.42 and 0.66 , respectively), which implies an improvement of $37 \%$. The IIC algorithm do not work as well as LCM, and $\mathrm{AE}$ makes the uncorrected case even worse.

In the ROC curve (Fig. 6), the improvement of our algorithm is very substantial whereas the IIC algorithm, which yields the best alternative results, hardly outperforms the original images.

This is probably because our method rejects the overexposed regions of the $\mathrm{BG}$, making our algorithm more robust against highlights. Furthermore, the $\mathrm{BG}-\mathrm{FG}$ relationship is more reliable than the transformation values calculated by $\mathrm{AE}$ and IIC algorithms, as the $\mathrm{BG}-\mathrm{FG}$ relationship has been trained for local rather than global illumination changes.

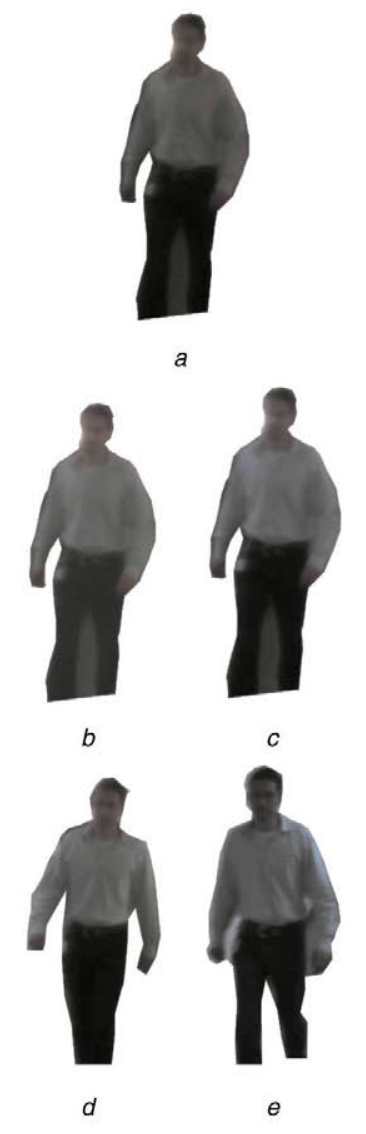

Fig. 5 Example of image correction

(a) Original image to be corrected. Corrected images for the intra-camera (b) and inter-camera $(c)$ results. Reference images taken in the intra-camera $(d)$ and inter-camera $(e)$ experiment. Images $(b)$ and $(c)$ are more similar to images $(d)$ and $(e)$ in colour distribution, respectively
Table 3 Results of cameras correction

\begin{tabular}{lcc}
\hline Method & MEC & Improvement, \% \\
\hline (a) Camera 1 correction & & \\
uncorrected & 0.66 & - \\
AE [3] & 0.69 & -4 \\
IIC [27] & 0.64 & 4 \\
AE + IIC [3, 27] & 0.67 & -1 \\
LCM & 0.42 & 37 \\
LCM $(-30,+60)$ & 0.50 & 24 \\
LCM $(-90,+200)$ & 0.55 & 18 \\
(b) Camera 2 correction & & \\
uncorrected & 0.59 & \\
AE [3] & 0.39 & 34 \\
IIC [27] & 0.70 & -19 \\
AE + IIC [3, 27] & 0.57 & 4 \\
LCM & 0.40 & 32 \\
LCM $(-30,+60)$ & 0.40 & 32 \\
LCM $(-90,+200)$ & 0.38 & 36 \\
(c) Comparing images between both cameras & \\
uncorrected & 0.69 & - \\
AE [3] & 0.61 & 12 \\
IIC [27] & 0.71 & -4 \\
AE + IIC [3, 27] & 0.66 & 5 \\
LCM & 0.50 & 27 \\
LCM $(-30,+60)$ & 0.52 & 24 \\
LCM (-90, +200) & 0.69 & -1 \\
\hline &
\end{tabular}

Value of the MEC besides the improvement compared with the images with no correction are shown. Each row is: uncorrected images, by using the $A E$ algorithm (AE), the IIC, $A E$ plus IIC $(A E+I I C)$ and our algorithm using three different segmentations for the FG: (i) manual - LCM - (ii) modifying the area size of the manual segmentations in -30 and $60 \%-\operatorname{LCM}(-30,+60)-$ and (iii) area variations of -90 and $200 \%-\operatorname{LCM}(-90,+200)$

The results in camera 2 confirm that LCM outperforms the others. Note that IIC does not improve the uncorrected case [in Table 3(b)] and - in comparison with (AE) alone - it

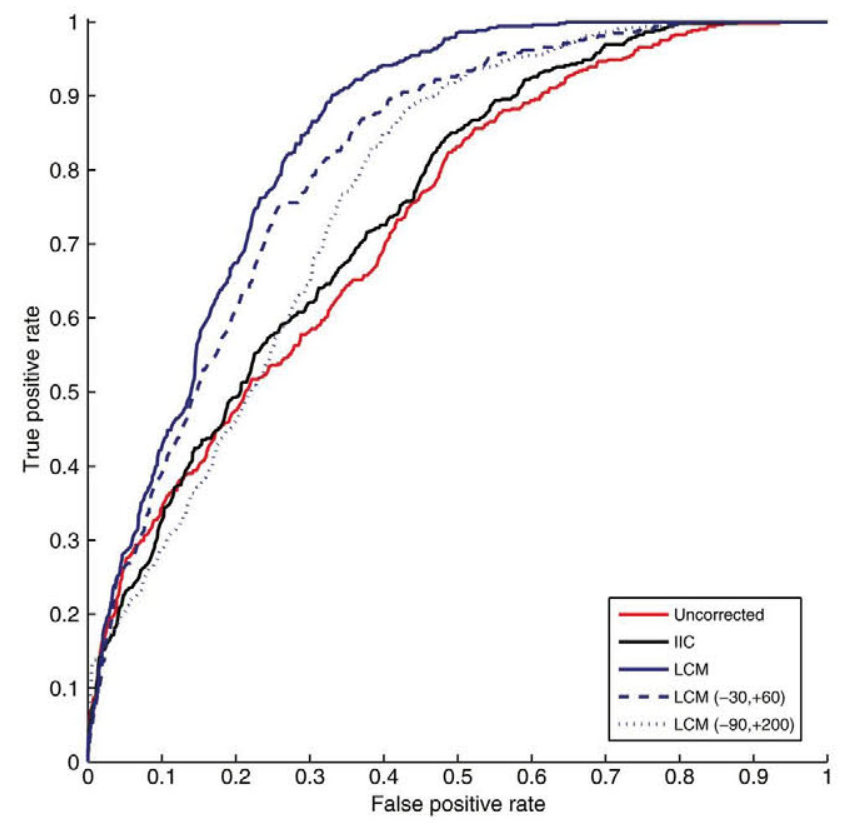

Fig. 6 ROC curve for camera 1

True positive rate against the false positive rate of the five analysis cases are shown. The lightest solid lines belong to the uncorrected case, the black solid lines to the best alternative performance (IIC [27]), the middle grey solid lines to our algorithm using a manual segmentation, the dashed ones to our algorithm with segmentation errors of -30 and $+60 \%$ of area variation and the dotted ones to our algorithm with segmentation errors of -90 and $200 \%$ 
Table 4 Estimated ICCM parameters using to correct images from camera 1

\begin{tabular}{lcc}
\hline Channel & $\alpha$ & $\beta$ \\
\hline red & 1.1909 & -0.0118 \\
green & 1.2043 & -0.0150 \\
blue & 1.3264 & -0.0195 \\
\hline
\end{tabular}

Intensity range is $[0,1]$

also does not improve when both methods $(\mathrm{AE}+\mathrm{IIC})$ are combined. Although results of $\mathrm{AE}$ are positive and noteworthy (MEC is 0.39 and the improvement is $34 \%$ ), $\mathrm{LCM}$ error is similar (MEC is 0.40 and the improvement is $32 \%)$.

In the case of camera 2, the most important source of light is the sun, and the indoor lights have less influence. Although indoor lights have been switched on/off, the influence of the outdoor light variation produces a higher intensity and colour variation. Thus, it seems that IIC is not able to estimate the correct main illuminant in every picture. In addition, the indoor lights do not over expose the image acquisition as happened in camera 1 . It can be said that the multiple light sources and overexposed $\mathrm{BG}$ areas in camera 1 make a more complex scenario compared with camera 2. This makes photometric variations in camera 2's FG objects simpler, so it seems reasonable that errors in uncorrected images are slightly smaller than in camera 1 , as Table 3(b) shows.

To conclude, results on this dataset demonstrate that our algorithm is able to correct photometric variations in objects even in complex illumination scenarios on condition that $\mathrm{BG}$ areas are close enough to $\mathrm{FG}$ objects and the camera works on the linear region. In this context, complex scenarios may be those where there are a multitude of reflections on Lambertian surfaces, at least one illuminant is changed and the intensity of lights vary significantly.

\subsection{Inter-camera results}

An example of a corrected image for the inter-camera correction is shown in Figs. $5 a, c$ and $e$. The estimates of the ICCM are shown in Table 4.

Table 3(c) shows the results of inter-camera correction. Compared with intra-camera results the MEC are slightly worse than the corresponding mean of cameras 1 and 2 MEC. Deterioration of LCM is probably because of different illuminations and shadow geometries at both cameras. Still our algorithm significantly outperforms the others. As Table 3(c) shows, the MEC improvement of IIC correction is negative and using the AE + IIC algorithm is fairly close to the uncorrected case (a difference of 5\%) because of the poor performance of camera 1. The inter-camera results show that LCM obtains a large improvement of $27 \%$, which it is noteworthy and more than twice the $\mathrm{AE}$ algorithm's improvement. Fig. 8 confirms the LCM improvement: although AE curve is over the uncorrected curve for almost every false positive rate value, the LCM curve is even closer to 1 and, hence, much better than AEs.

These results show that in a non-overlapping multi-camera system - although scenarios' illumination is different (indoor and outdoor) - our algorithm is able to estimate a correction that helps to maintain the colour appearance of objects in such scenarios.

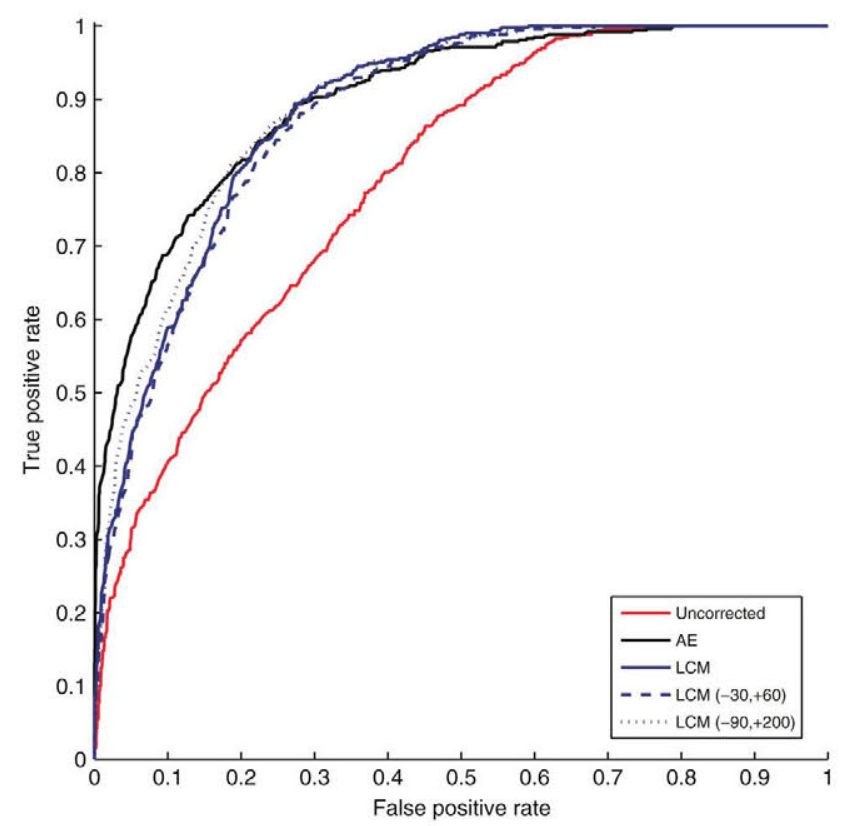

Fig. 7 ROC curve for camera 2

Figure description is similar to Fig. 6 . In this case, the best alternative is the $\mathrm{AE}[3]$

\subsection{Robustness against segmentation errors}

To evaluate the robustness of our method against segmentation errors in the FG extraction, an additional experiment was carried out.

The size of each FG object was extended or reduced. The values of $-30 / 60 \%$ and $-90 / 200 \%$ were chosen to reflect small and large errors in the FG segmentation, where a negative value refers to a reduction and a positive value refers to an extension. For each image, the decision about the extension or reduction was random.

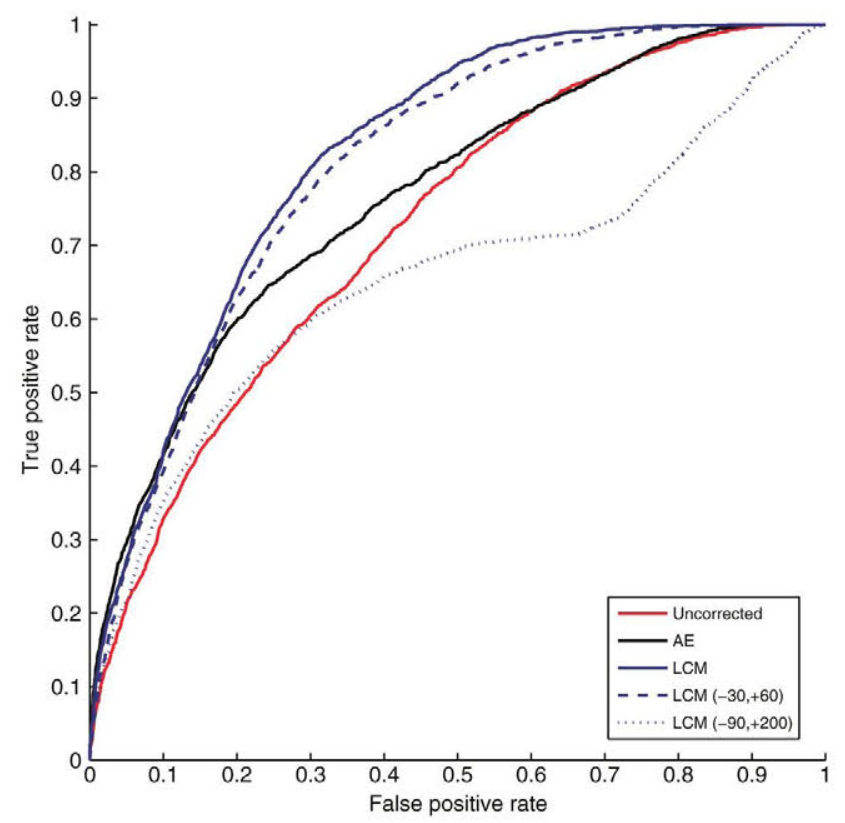

Fig. 8 ROC curve for both cameras

Figure description is similar to Fig. 7. In this case, the best alternative is the $\mathrm{AE}[3]$ 
Note that the scope of this paper is on colour correction. For colour correction, the FG segmentation is only used during training mode whereas the correction during run-time is based on $\mathrm{BG}$ intensities only. FG segmentation during run-time only is used for evaluation. To determine the sensitivity of the colour correction method, only the segmentation during training mode is modified by erosions and dilations, and not the segmentation used in evaluation within the EMD matching.

The assessment was the same as the exposed above. The results are presented in Table 3 and Figs. 6-8 for comparison with the rest of the methods. These demonstrate that our method maintains similar error rates when the size variation is reduced by $30 \%$ and enlarged by $60 \%$. In this case it still outperforms the AE, IIC and $\mathrm{AE}+\mathrm{IIC}$ methods. The deterioration of the algorithm becomes noticeable when the segmentation errors are always large (in the case of -90 and $200 \%$ ).

\section{Conclusions}

In this paper, we describe a computationally efficient method to correct photometric variations between scenarios. For each image, these variations are estimated by simple vector operations and the correction is based on a double linear mapping. This is linked with the fact that, contrary to other colour constancy algorithms, the illuminants do not have to be estimated. Therefore, the scenarios are easy to calibrate and the use of canonical, known illuminants or colour-checker patterns are not required.

The main conclusions of this paper are the following: our algorithm performs much better than uncorrected images and state-of-the-art AE together with a representative colour correction algorithm. Furthermore, it is able to work properly in complex environments with several illuminants because, unlike most of inter-camera and other correction methods, local photometric changes are taken into account and the estimate of the BG-FG relation makes it easy to adapt changes in any object using BG information. It is also robust against FG segmentation errors.

Our algorithm is able to correct changes in the settings of the camera as long as the change in the camera response is linear. Gain, aperture and exposure time variations mostly produce linear variations in the image if the post-processing is also linear. In future work, we will investigate a combination of non-linear in-camera processing as present in many cameras with our algorithm. This shall include automatic characterisation of such non-linear processing [31-33] and an adequate compensation.

Future work will also involve the development of a segmentation algorithm that automatically creates a segmentation of $\mathrm{BG}$ regions that are classified by their response to the illumination changes.

\section{References}

1 Wang, Y.K., Wang, C.F.: 'Face detection with automatic white balance for digital still camera'. Int. Conf. Intelligent Information Hiding and Multimedia Signal Processing, Pasadena, CA, USA, 2008, pp. 173-178

2 Park, S., Kim, G., Jeon, J.: 'The method of auto exposure control for low-end digital camera'. Eleventh Int. Conf. Advanced Communication Technology, Gangwon-Do, Korea (South), 2009, vol. 03, pp. 1712-1714

3 Liu, J., Ren, D., Zou, J., Wu, Y., Li, S.: 'Study of automatic exposure algorithm based on HD IP camera'. Int. Conf. Advanced Intelligence and Awareness Internet, Beijing, China, 2010, pp. 265-268

4 Bouma, H., Borsboom, S., den Hollander, R., Landsmeer, S., Worring, M.: 'Re-identification of persons in multicamera surveillance under varying viewpoints and illumination'. Proc. Society of Photo-Optical Instrumentation Engineers (SPIE), 2012, vol. 8359 , pp. 83590Q-83590QH10

5 Barnard, K., Martin, L., Funt, B.V.: 'Colour by correlation in a three-dimensional colour space'. European Conference on Computer Vision ECCV (1), Dublin, Ireland, 2000, pp. 375-389

6 Hsu, E., Mertens, T., Paris, S., Avidan, S., Durand, F.: 'Light mixture estimation for spatially varying white balance'. ACM SIGGRAPH 2008 Papers, SIGGRAPH '08, New York, NY, USA, 2008, pp. 70:1-70:7

7 Gijsenij, A., Gevers, T., van de Weijer, J.: 'Computational color constancy: survey and experiments', IEEE Trans. Image Process., 2011, 20, (9), pp. 2475-2489

8 Riess, C., Eibenberger, E., Angelopoulou, E.: 'Illuminant color estimation for real-world mixed-illuminant scenes'. 2011 IEEE Int. Conf. Computer Vision Workshops (ICCV Workshops), Barcelona, Spain, 2011, pp. 782-789

9 Wannous, H., Lucas, Y., Treuillet, S., Mansouri, A., Voisin, Y. 'Improving color correction across camera and illumination changes by contextual sample selection', J. Electron. Imaging, 2012, 21, (2), 023,015-1-023,015-14

10 Grossberg, M., Nayar, S.: 'Modeling the space of camera response functions', IEEE Trans. Pattern Anal. Mach. Intell, 2004, 26, (10), pp. $1272-1282$

11 Withagen, P.J., Schutte, K., Groen, F.C.A.: 'Global intensity correction in dynamic scenes', Int. J. Comput. Vis., 2010, 86, (1), pp. 33-47

12 Parameswaran, V., Singh, M., Ramesh, V.: 'Illumination compensation based change detection using order consistency'. IEEE Computer Society Conf. Computer Vision and Pattern Recognition, San Francisco, CA, USA, 2010, pp. 1982-1989

13 Sayed, M.S., Delva, J.G.R.: 'An efficient intensity correction algorithm for high definition video surveillance applications', IEEE Trans. Circuits Syst. Video Technol, 2011, 21, (11), pp. 1622-1630

14 Porikli, F.: 'Inter-camera color calibration by correlation model function'. 2003 Int. Conf. Image Processing, Barcelona, Spain, 2003, vol. 2, pp. 133-136

15 Javed, O., Shafique, K., Shah, M.: 'Appearance modeling for tracking in multiple non-overlapping cameras'. IEEE Computer Society Conf Computer Vision and Pattern Recognition, San Diego, CA, USA, 2005, vol. 2, pp. 26-33

16 Madden, C., Cheng, E., Piccardi, M.: 'Tracking people across disjoint camera views by an illumination-tolerant appearance representation', Mach. Vis. Appl, 2007, 18, (3), pp. 233-247

17 Gilbert, A., Bowden, R.: 'Incremental, scalable tracking of objects inter camera', Comput. Vis. Image Underst., 2008, 111, (1), pp. 43-58

18 Prosser, B., Gong, S., Xiang, T.: 'Multi-camera matching using bi-directional cumulative brightness transfer functions'. British Machine Vision Conference, Leeds, UK, 2008

19 D'Orazio, T., Mazzeo, P., Spagnolo, P.: 'Color brightness transfer function evaluation for non overlapping multi camera tracking'. Third ACM/IEEE Int. Conf. Distributed Smart Cameras, Como, Italy, 2009, vol. 2, pp. 1-6

20 Loy, C.C., Xiang, T., Gong, S.: 'Multi-camera activity correlation analysis'. IEEE Computer Society Conf. Computer Vision and Pattern Recognition, Miami, Florida, USA, 2009, pp. 1988-1995

21 Siebler, C., Bernardin, K., Stiefelhagen, R.: 'Adaptive color transformation for person re-identification in camera networks'. Fourth ACM/TEEE Int Conf. Distributed Smart Cameras, Atlanta, GA, USA, 2010, pp. 199-205

22 Chen, K.W., Lai, C.C., Lee, P.J., Chen, C.S., Hung, Y.P.: 'Adaptive learning for target tracking and true linking discovering across multiple non-overlapping cameras', IEEE Trans. Multimedia, 2011, 13, (4), pp. 625-638

23 Gijsenij, A., Lu, R., Gevers, T.: 'Color constancy for multiple light sources', IEEE Trans. Image Process., 2012, 21, (2), pp. 697-707

24 Finlayson, G.D., Drew, M.S., Funt, B.V.: 'Spectral sharpening: sensor transformations for improved color constancy', J. Opt. Soc. Am. A, 1994, 11, (5), pp. 1553-1563

25 Bouwmans, T.: 'Recent advanced statistical background modeling for foreground detection-a systematic survey', Recent Patents Comput. Sci., 2011, 4, (3), pp. 147-176

26 Rubner, Y., Tomasi, C., Guibas, L.J.: 'The Earth Mover's Distance as a metric for image retrieval', Int. J. Comput. Vis., 2000, 40, (2), pp. 99-121

27 Tan, R.T., Nishino, K., Ikeuchi, K.: 'Illumination chromaticity estimation using inverse-intensity chromaticity space'. Proc. IEEE Computer Society Conf. Computer Vision and Pattern Recognition, 2003, Madison, Wisconsin, USA, 2003, pp. I-673-I-680

28 Withagen, P.J., Groen, F.C.A., Schutte, K.: 'CCD color camera characterization for image measurements', IEEE Trans. Instrum. Meas., 2007, 56, (1), pp. 199-203

29 Kim, S.J., Lin, H.T., Lu, Z., Susstrunk, S., Lin, S., Brown, M.S.: 'A new in-camera imaging model for color computer vision and its application', IEEE Trans. Pattern Anal. Mach. Intell., 2012, 34, (12), pp. 2289-2302 
30 Huber, P.J.: 'Robust statistics. Wiley series in probability and statistics' (Wiley-Interscience, 1981)

31 Goldman, D.: 'Vignette and exposure calibration and compensation', IEEE Trans. Pattern Anal. Mach. Intell., 2010, 32, (12), pp. $2276-2288$
32 Farid, H.: 'Blind inverse gamma correction', IEEE Trans. Image Process., 2001, 10, (10), pp. 1428-1433

33 Huang, S.C., Cheng, F.C., Chiu, Y.S.: 'Efficient contrast enhancement using adaptive gamma correction with weighting distribution', IEEE Trans. Image Process., 2013, 22, (3), pp. 1032-1041 